\title{
Identifying Prognostic Indicators for Electrical Treeing in Solid Insulation through PD Analysis
}

\author{
N.H. Aziz*, M.D. Judd, V.M. Catterson \\ University of Strathclyde, Glasgow, U.K \\ *hakimah.aziz@strath.ac.uk
}

\begin{abstract}
This paper presents early results from an experimental study of electrical treeing on commercially available pre-formed silicone samples. A needle-plane test arrangement was set up using hypodermic needle. Partial discharge (PD) data was captured using both the IEC60270 electrical method and radio frequency (RF) sensors, and visual observations are made using a digital microscope. Features of the PD plot that corresponded to electrical tree growth were assessed, evaluating the similarities and differences of both PD measurement techniques. Three univariate phase distributions were extracted from the partial discharge phase-resolved (PRPD) plot and the first four statistical moments were determined. The implications for automated lifetime prediction of insulation samples due to electrical tree development are discussed.
\end{abstract}

Keywords-Silicone rubber (SiR); electrical treeing; partial discharge; prognostics.

\section{INTRODUCTION}

Prognostic is defined in [1] as "the prediction of the future state of health based on current and historical health conditions". In solid insulation, the ultimate goal of prognostics is to predict the advent of failure, i.e., insulation breakdown, in terms of remaining useful life (RUL). This paper takes a step towards a prognostic system for insulation breakdown, by investigating features in data which correspond to insulation ageing.

Electrical treeing is a common degradation mechanism in solid insulation with progressive faults leading to potentially catastrophic failure. Electrical treeing can be initiated from a water tree or insulation defects that can give rise to large local field concentration. Such defects are metallic protrusions, micro voids and contaminants [2].

The presence of electrical treeing can be detected through partial discharge (PD) monitoring [3]. Automated PD data analysis systems have been shown to correctly diagnose the defect causing PD [4]. This paper aims to extend this concept towards predicting the evolution of the defects by investigating the features of the PD plot which correspond to electrical tree growth; moving from diagnostics (identifying the presence of a tree) to prognostics.

\section{EXPERIMENTAL SETUP}

Various polymers have been used in electrical treeing experiments, e.g. epoxy resin, cross-linked polyethylene (XLPE), ethylene vinyl acetate (EVA), and silicone rubber (SiR). In this paper, SiR is chosen as the test specimen as it requires a very short time to initiate electrical treeing even at low applied voltage.

This preliminary study uses commercially available preformed silicone samples, which ensure consistency and eliminate the need for mixing, degassing and heating in sample preparation. The sample is tested using a needle-plane arrangement by inserting a fresh hypodermic needle $(50 \mathrm{~mm}$ length, $1 \mathrm{~mm}$ diameter, $10 \mu \mathrm{m}$ tip radius) into the sample with $2 \mathrm{~mm}$ gap between the needle tip and the plane electrode. The needle is pulled slightly backward to create a needle-shaped void. The sample is placed against the earth plate inside an acrylic container and immersed in mineral oil to suppress extraneous PD. The dimension of the test sample is illustrated in Fig 1.

Both IEC60270 and radio frequency (RF) detection methods were applied for PD monitoring. The phase-resolved partial discharge (PRPD) patterns from both techniques were recorded through computer aided PD measuring system, i.e. LDS-6 (Doble Lemke) and PortSUB (Qualitrol) respectively. The apparent charge magnitude from LDS-6 is recorded manually every 5 minutes followed by a 2 -minute downtime. PortSUB on the other hand, records the corresponding level of detected signal automatically in 64 phase buckets per cycle for 255 cycles. The growth of electrical treeing is monitored simultaneously using a digital microscope (AM2011 Dino-Lite Basic) and the image of the test sample is captured every 5 minutes.

\section{RESULTS AND DISCUSSION}

This paper reports results from one particular electrical treeing experiment in a pre-formed SiR sample. This sample was chosen for detailed study, as the morphology of the tree corresponds with other experiments reported in the literature. The test sample was aged for 18 hours and the growth evolution is highlighted below by referring to the voltage application regime outlined in Fig. 2. The voltage was periodically switched off as the experiment could not be left unattended, accepting that this may influence the tree growth. 


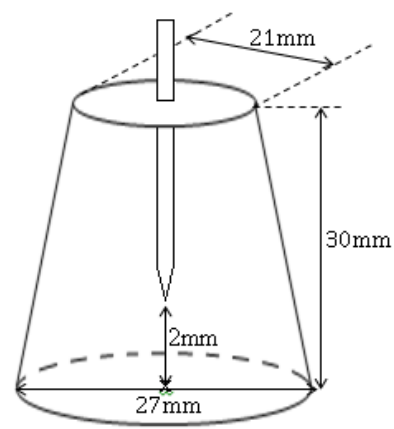

Fig. 1. Test sample.

\section{A. Optical Observation}

Stage A: Fig. 3a shows the image from the digital microscope before the experiment starts (point A0). First, ramp voltage was applied to the sample at $0.3 \mathrm{kV} / \mathrm{min}$ starting from $2.9 \mathrm{kV}$ (the initial voltage of the supply). After 13 minutes (point A1), the PD started to show higher values than background noise level. At this point, it is assumed that an electrical tree is initiated giving the partial discharge inception voltage (PDIV) of $7.1 \mathrm{kV}$. However, it is difficult to detect the electrical tree from the captured image shown in Fig 3b. In order to speed up the tree growth, the applied voltage was increased to a value marginally above PDIV, i.e. $8 \mathrm{kV}$.

Stage B: Constant voltage of $8 \mathrm{kV}$ was applied at this stage for nearly 5 hours to investigate the tree propagation behavior. Initially, the tree growth rate is very low at about $1.8 \mu \mathrm{m} / \mathrm{min}$ (measure of the tree length from the needle tip). Only after 4 hours (point B1), the PD values showed significant changes in magnitude and number of occurrence as the tree grew into a bush shape (Fig. 3c).

Stage C: The effect of higher voltage on the existing tree shape was observed by increasing the voltage to $9 \mathrm{kV}$. After 26 minutes (point C1), a new tree started to grow from the needle tip as indicated by the darker point in Fig 3d. About an hour later (point C2), the tree became a trunk when it started to form two branches as illustrated in Fig. 3e. The first branch that reaches the earth plate (Fig. 3f) was at point $\mathbf{C 3}$ (after 4.5 hours) with $8 \mu \mathrm{m} / \mathrm{min}$ growth rate which is greater than Stage B. However, the breakdown did not occur and more branches were created after that, e.g. at point $\mathbf{C 4}$. The same result was reported in [5] with the assumption that the current is too low to cause breakdown. Over time, the applied optical observation became less reliable as the tree grew at every angle which requires more than one camera to capture accurate images.

\section{B. PRPD Pattern}

This paper employed the combination of IEC60270 and RF technique as recommended in [6] as a potential successful diagnostic tool. Fig. 4 shows the PRPD pattern from the IEC60270 measurements corresponding to the RF technique in Fig. 5 for events occurred at points B1, C3 and C4 of Fig. 2. Overall, the RF technique gives the fairly similar PRPD pattern for all three growth points.

In this experiment, electrical treeing was first observed when PD measurement showed an increase in both the amplitude and repetition rate. These values, however, fluctuated throughout the experiment depending on electrical treeing activity. From observation, when the PRPD showed a very high repetition rate, the recorded images showed a significant change in tree growth regardless of the maximum value of the discharges. The high repetition rate is associated with high levels of PD activity $(50 \mathrm{pC}-300 \mathrm{pC})$ when a bush type tree is detected or an electrical tree is initiated from the needle tip (as described in Section IIIA for points A1, B1 and $\mathrm{C} 1$ of Fig. 2). For instance, the bush type tree in Fig. 3c produced the simultaneous PD pattern of Fig. $4 \mathrm{a}$ and $5 \mathrm{a}$ with a maximum discharge greater than $100 \mathrm{pC}$. In a different case, the creation of tree branches from the existing trunk at point $\mathrm{C} 4$ of Fig. 2 results in a high number of PD occurrences but lower maximum discharge, i.e. less than $15 \mathrm{pC}$ as shown in Fig. $4 \mathrm{c}$ and $5 \mathrm{c}$. As the tree branches stop growing when the branches touch the earth plate, fewer PD pulses are detected. The first branch to touch the ground is at point $\mathrm{C} 3$ and the PRPD patterns are depicted in Fig. $4 \mathrm{~b}$ and $5 \mathrm{~b}$ with apparent charges lower than $15 \mathrm{pC}$.

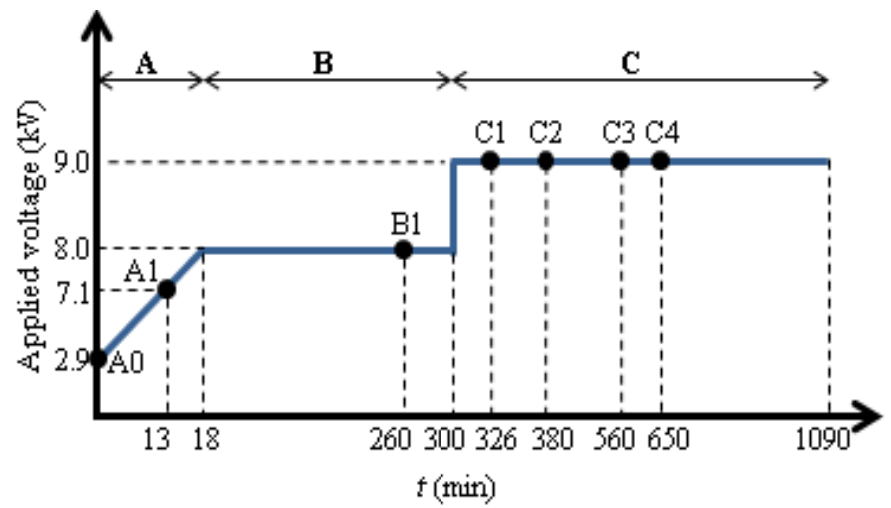

Fig. 2. Voltage application regime for electrical treeing experiment.

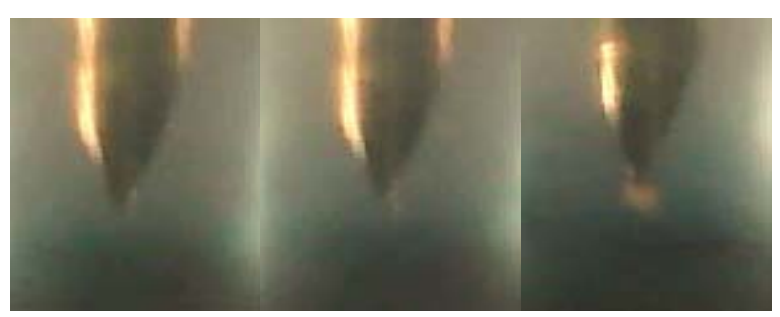

(a)

(b)

(c)

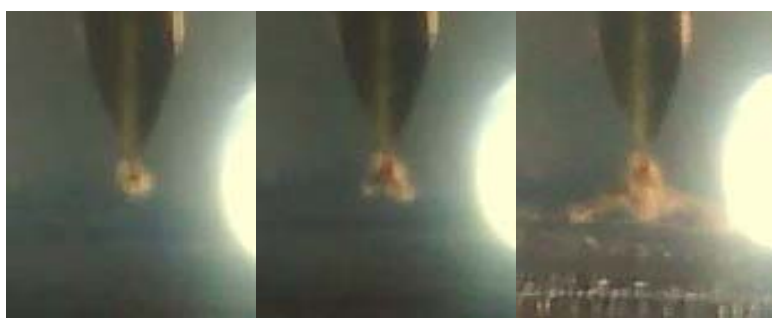

(d)

(e)

(f)

Fig. 3. Tree images captured from digital microscope at points of (a) A0, (b) $\mathrm{A} 1$, (c) $\mathrm{B} 1$, (d) $\mathrm{C} 1$, (e) $\mathrm{C} 2$ and (f) $\mathrm{C} 3$ referring to Fig. 2 


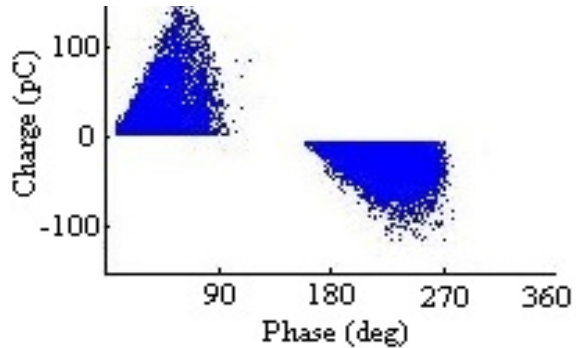

(a)

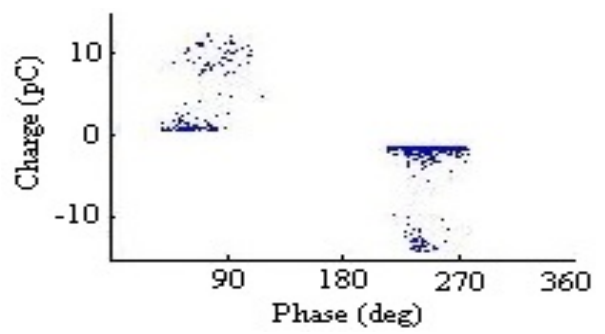

(b)

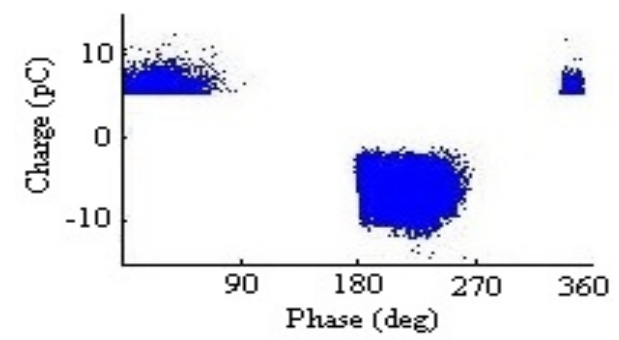

(c)

Fig. 4. PRPD patterns measured with IEC60270 standard at points of (a) B1, (b) $\mathbf{C 3}$ and (c) $\mathbf{C 4}$ referring to Fig. 2.

\section{Statistical Analysis}

In order to identify features of the PD plot corresponding to the tree growth, three univariate phase distributions are extracted from the PRPD patterns of both PD measurement techniques: Pulse number distribution, $H_{n}(\varphi)$, maximum pulse height distribution, $H_{q m}(\varphi)$, and mean pulse height distribution $H_{q n}(\varphi)$. Four statistical moments of mean, $\mu$, standard deviation, $\sigma$, skewness, $S k$, and kurtosis, $K u$, are calculated for the positive and negative half cycles of those distributions [7].

A number of features from the IEC60270 and the RF technique (Fig. 6 and 7 respectively) have been identified corresponding to the tree growth:

1) The mean and standard deviation of positive and negative half cycles are symmetrical for all three distributions in the IEC60270 data: This feature had been discussed in detail in [8]. However, for RF technique, only the $H_{n}(\varphi)$ distribution is symmetrical.

2) The mean and standard deviation of $H_{n}(\varphi)$ distribution increase in absolute value as the sample ages: The number of pulses per cycle is generally increasing with the size of the tree, but also the number of pulses per cycle varies more from cycle to cycle as the insulation ages. When comparing this

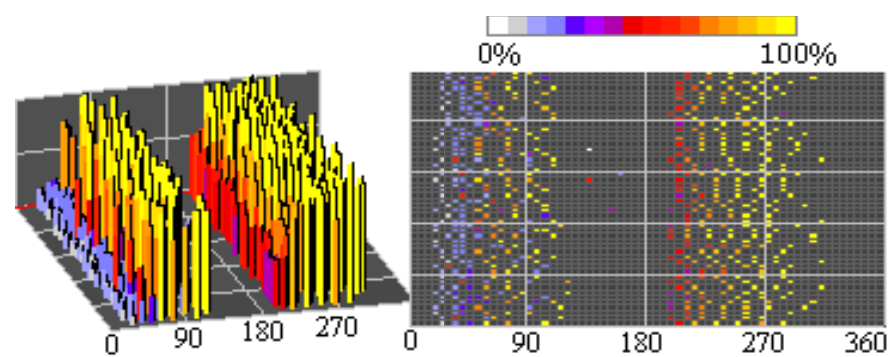

(a)

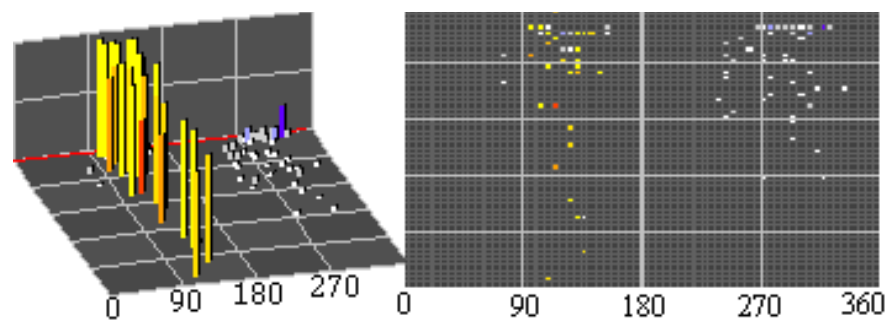

(b)

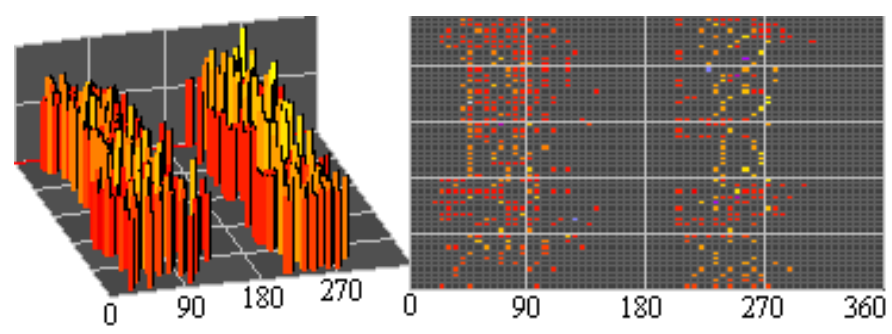

(c)

Fig. 5. PRPD patterns measured with RF technique at points of (a) B1, (b) $\mathbf{C} 3$ and (c) $\mathbf{C 4}$ referring to Fig. 2.

feature between IEC60270 and RF technique, the former technique has a wider range of pulse numbers because of the difference in data range explained in Section II.

3) Skewness of all three distributions tend to change from positive to negative: As the tree grows, the peaks of $\mathrm{PD}$ distributions tend to move from the start of one half cycle to the nearer the end. This is clearest in the $H_{n}(\varphi)$ distributions of the RF technique, which transitions from positive to negative skew as the sample ages.

4) Kurtosis of $H_{q m}(\varphi)$ and $H_{q n}(\varphi)$ distributions is almost zero: Most of the time, the number of pulses clusters fairly close to the center of the half cycle.

\section{CONCLUSION AND FUTURE WORK}

Identifying prognostic indicators is the first step in predicting RUL of the insulation system. Those indicators are identified in this paper through statistical analysis on $H_{n}(\varphi)$, $H_{q m}(\varphi)$, and $H_{q n}(\varphi)$ distributions. However, not all features listed in Section III(C) will be considered in lifetime prediction. As electrical treeing is a degradation process which evolves over time, thus, only features that have significant characteristics over time are chosen, i.e. mean and standard deviation of $H_{n}(\varphi)$ and skewness of $H_{n}(\varphi), H_{q m}(\varphi)$, and $H_{q n}(\varphi)$. 
Both IEC60270 and RF technique show fairly similar PRPD patterns. From statistical analysis, the RF technique shows larger variation because of the reduced phase resolution. This work will continue the identification of prognostic indicators using other promising methods, e.g. pulse shape analysis and pulse sequence analysis. In future, these features will be used for lifetime prediction of insulation samples.

\section{REFERENCES}

[1] N. M. Vichare and M. G. Pecht, "Prognostics and health management of electronics," IEEE Transactions on Components and Packaging Technologies, vol. 29, no. 1, pp. 222-229, 2006.

[2] R. Bozzo, F. Guastavino, and G. C. Montanari, "Electrical tree tests. Probabilistic inference and insulating material evaluation," IEEE Transactions on Dielectrics and Electrical Insulation, vol. 5, no. 5, pp. 734-740, 1998.

[3] Suwarno et al., "Partial discharge patterns of electrical treeing in polyethylene," Proceedings of the 4th International Conference on Properties and Applications of Dielectric Materials, vol. 1, pp. 379-382, 1994.
[4] E. Gulski, P. Morshuis, and F. Kreuger, "Conventional and timeresolved measurements of partial discharges as a tool for diagnosis of insulating materials," Proceedings of the 4th International Conference on Properties and Applications of Dielectric Materials, vol. 2, pp. 666-669, 1994.

[5] R. Vogelsang, B. Fruth, T. Farr, and K. Fr hlich, "Detection of electrical tree propagation by partial discharge measurements," European Transactions on Electrical Power, vol. 15, no. 3, pp. 271-284, 2005.

[6] A. J. Reid, M. Judd, R. Fouracre, B. Stewart, and D. Hepburn, "Identification of simultaneously active partial discharge sources using combined radio frequency and IEC60270 measurement," Science, Measurement \& Technology, IET, vol. 5, no. 3, pp. 102-108, 2011.

[7] V. M. Catterson, S. Bahadoorsingh, S. Rudd, S. D. McArthur, and S. M. Rowland, "Identifying harmonic attributes from online partial discharge data," IEEE Transactions on Power Delivery, vol. 26, no. 3, pp. 1811$1819,2011$.

[8] S. J. Dodd, N. Chalashkanov, and J. C. Fothergill, "Statistical analysis of partial discharges from electrical trees grown in a flexible epoxy resin," Annual Report Conference on Electrical Insulation and Dielectric Phenomena, CEIDP, pp. 666-669, 2008
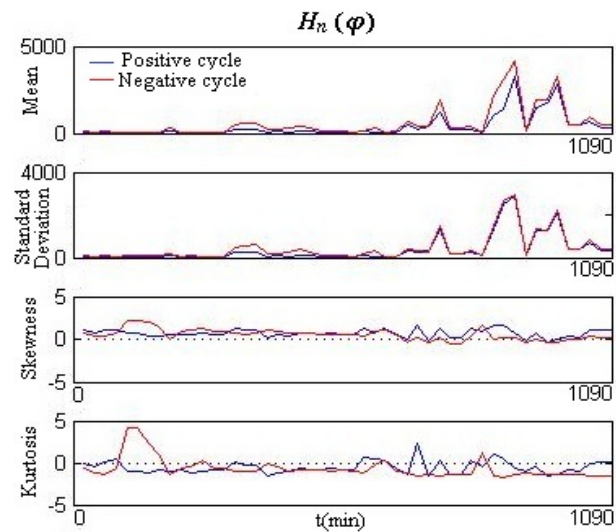

(a)
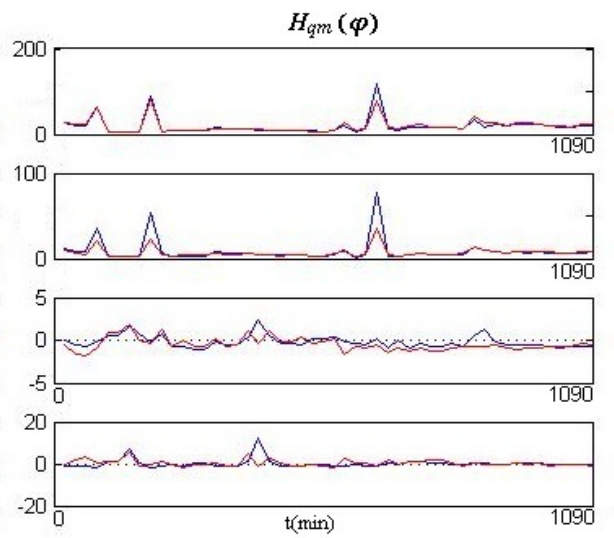

(b)
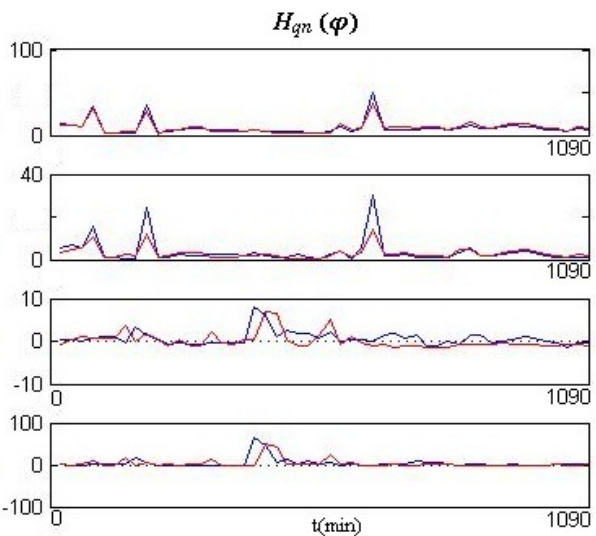

(c)

Fig. 6. Statistical features calculated from IEC60270 measurement (a) Pulse number distribution, $H_{n}(\varphi)$, (b) Mean pulse height distribution, $H_{q n}(\varphi)$, and (c) Maximum pulse height distribution, $H_{q m}(\varphi)$.
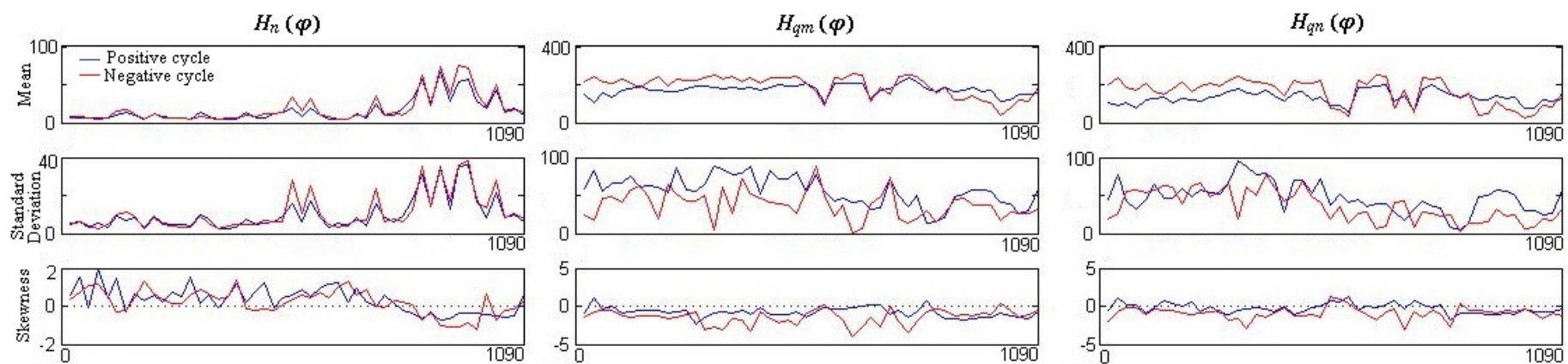

(a)

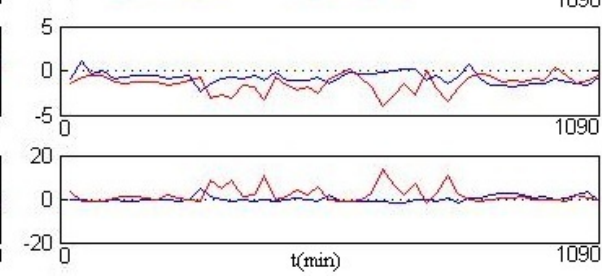

(b)

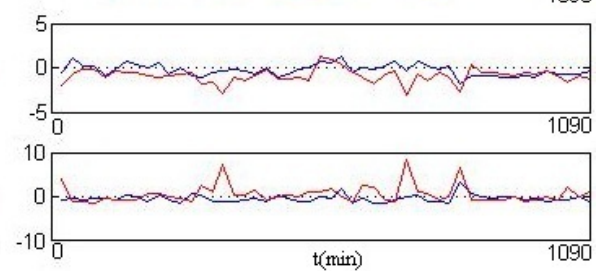

(c)

Fig. 7. Statistical features calculated from RF technique (a) Pulse number distribution, $H_{n}(\varphi)$, (b) Mean pulse height distribution, $H_{q n}(\varphi)$, and (c) Maximum pulse height distribution, $H_{q m}(\varphi)$. 